\title{
Compact laser probe for surface acoustic waves
}

\author{
V.V. Semenov, I.V. Blonskyi, V.G. Gryts' \\ Institute of Physics, NAS of Ukraine, \\ 46, prosp. Nauky, 03028 Kyiv, Ukraine
}

\begin{abstract}
Our compact laser probe for surface acoustic waves (SAW) is designed to study properties of SAW substrates and control technological processes of their manufacturing. These substrates are widely used in device manufacturing, controlling systems, consumer electronics, etc. In this probe, we use the Bragg modulator in the double-pass scheme, which provides its compactness and multifunctionality.
\end{abstract}

Keywords: laser probing, surface acoustic wave, Bragg modulator.

Manuscript received 06.10.09; accepted for publication 22.10.09; published online 30.12.09.

\section{Introduction}

Fields and tasks of application of substrates with excited surface acoustic waves (SAW) are very wide and continuously expanded [1 -6]. Commonly used facilities with SAW elements are as follows: frequency band filters, correlators, convolvers, delay lines and so on. This list constantly grows.

Sources for probing the substrates with a coherent light beams such as gas lasers are very bulky and inconvenient for application in compact SAW probes. Therefore, we use a semiconductor solid-state laser, which provides compactness and versatility of the offered probe. Composition and constitution of this device provides its stability against external factors, namely: increased temperature, vibration and shocks, acoustic signals, background illumination, etc.

\section{Functional scheme of the probe}

The requirements to compactness and operational stability are functionally realized in this probe. Fig. 1 shows the main elements of the functional scheme of our SAW probe. The laser illuminator 1 feeds the Bragg modulator 2 with a light beam. The diffraction beam of the zero order (forward pass) illuminates the SAW substrate and, being modulated with the SAW excitation frequency $f$, returns to the Bragg modulator. After the second pass through the Bragg modulator, the beam illuminates the photodetector 5. These two passes of the beam through the modulator provides the spectral shifts $\pm 2 f_{\mathrm{B}}$ to the signal of the SAW substrate with the frequency $f$. After heterodyning the signal with the frequency $2 f_{\mathrm{B}}$, the signal of the frequency $f$ is received and processed in a standard manner. Used in this probe liquid $(28 \mathrm{MHz})$ and solid-state $(60$ and $80 \mathrm{MHz})$ acoustic-and-optical modulators provide repeated heterodyning the received signals with the frequencies $56,120,160 \mathrm{MHz}$. The operational signals of the probe are reduced down to the range $400-500 \mathrm{kHz}$ where commonly used integrated circuits are applied. Mechanical elements for alignment of probe optical elements are traditional.

\section{Measurements with SAW substrates}

The data for measurements of SAW substrate properties were obtained using the prototype of compact laser probe for SAW under illumination of a substrate with a laser source of $5 \mathrm{~mW}$ power at the SAW excitation frequency $f=28 \mathrm{MHz}$. The substrate was made of lithium niobate with dimensions $40 \times 30 \times 0.3 \mathrm{~mm}^{3}$. The modulation frequency was $f_{\mathrm{B}}=14.2325 \mathrm{MHz}$ (we tuned the mixer at the lower frequency). Also, we used the standard amplifier of the intermediate frequency $f_{\text {if }}=465 \pm 5 \mathrm{kHz}$ and phase-meter $\Phi К 2-12$.

The plots of transversal amplitude and longitudinal phase profiles for the SAW on the tested substrate (Figs $2 a, b)$ show the presence of a non-uniformity (scratch or discontinuity flaw) on this substrate, which is clearly seen in the transversal amplitude profile. To improve information value needs $\mathrm{x}, \mathrm{y}$-scanning with a microobjective over the whole surface of the substrate with SAW as well as two-dimensional indication of these control data. The mentioned functions and measurement of the wave phase within the range $\pm \pi / 2$ should be provided by an integrated SAW controller. 


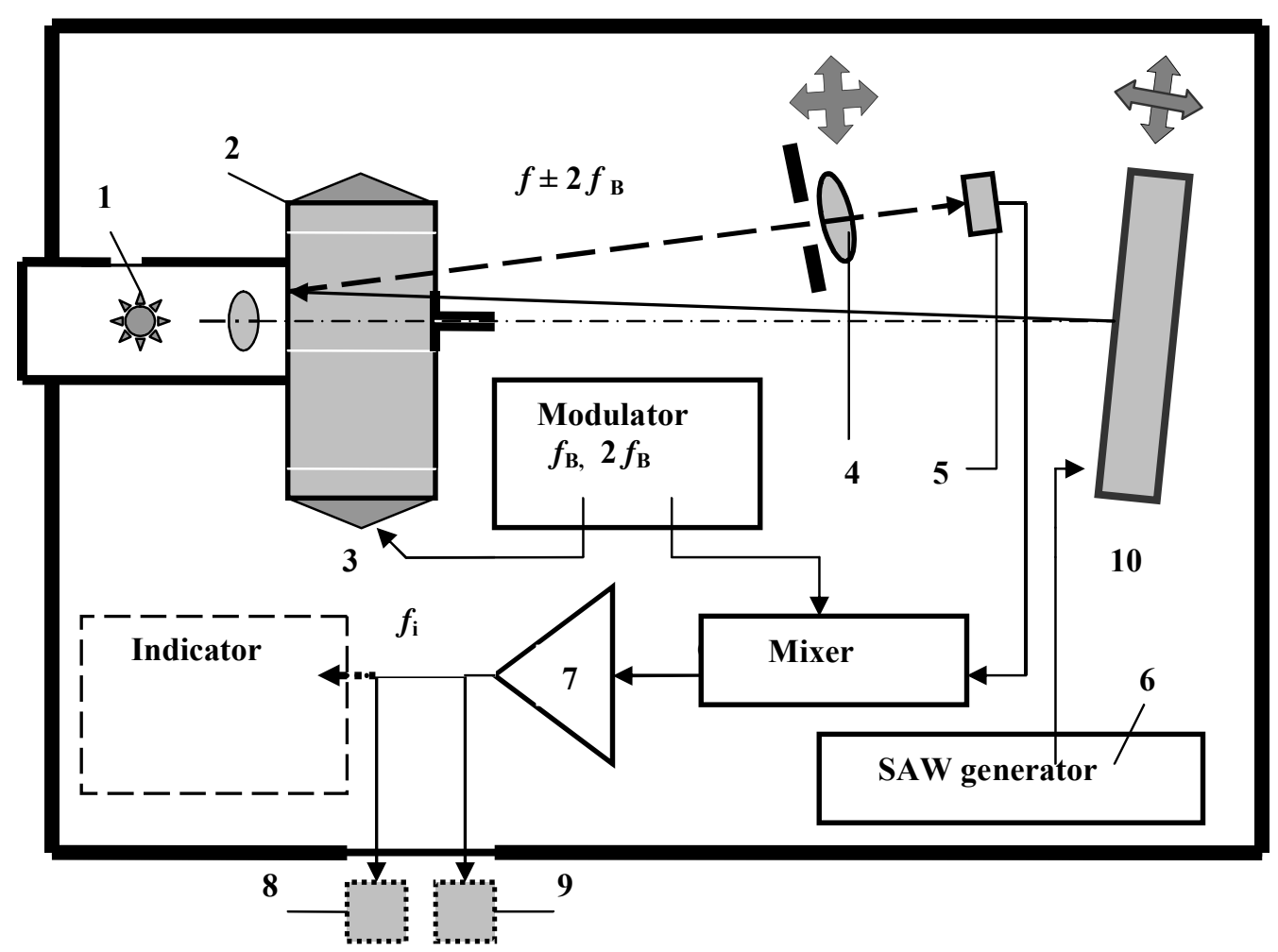

Fig. 1. Functional scheme of the compact SAW probe. 1 - laser, 2 - Bragg modulator, 3 - modulator excitation, 4 - objective, 5 - photodetector, 6 -SAW generator, 7 - intermediate frequency amplifier, 8 -amplifier, 9 - phase-meter, 10 - substrate. 8 and 9 are external facilities used for testing the probe.

A, rel. un.

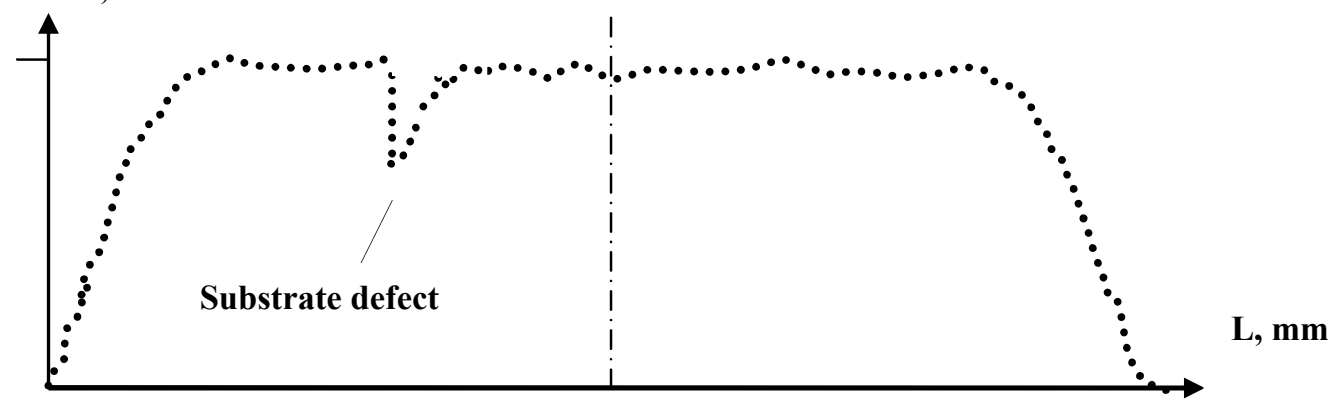

Fig. 2a. Transversal amplitude profile of SAW substrate vibrations.

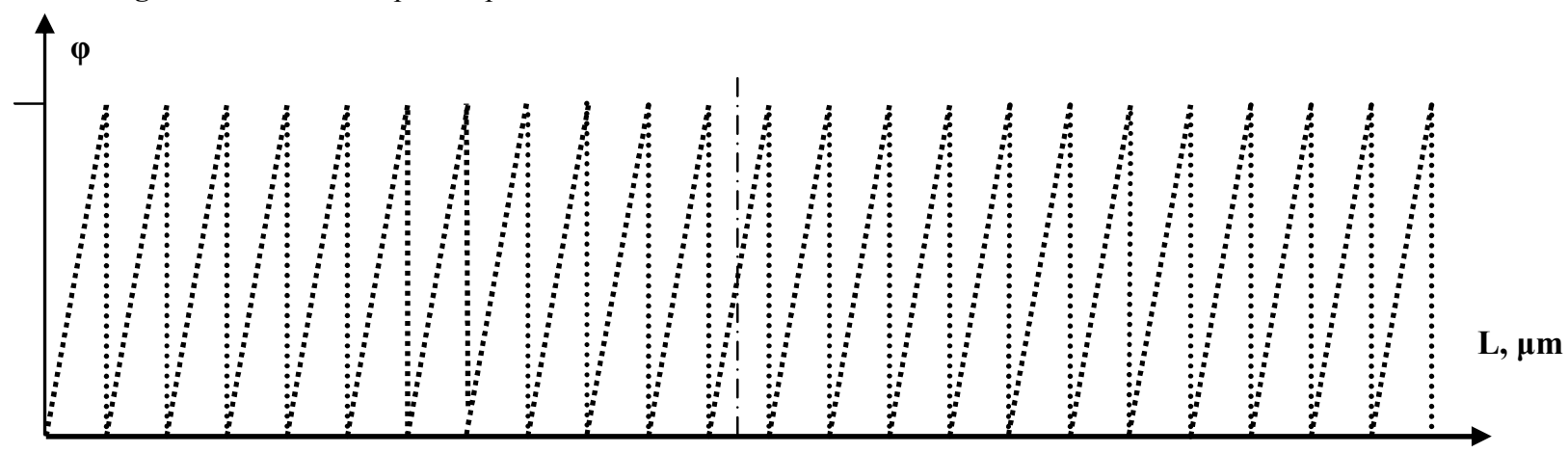

Fig. 2b. Longitudinal axial phase profile of SAW substrate vibrations. 


\section{Construction}

Compactness defined our main approaches to the probe design. There taken into account were design solutions for functional electronic elements - integrated circuits, optical elements - laser micro-optics, mechanic tuning facilities - laser micro-mechanics with controllers, plugin radiofrequency elements by way of integrated circuits or cases with connectors.

The case of the probe contains electronic units and components, elements of external communications, selfcontained power supply, connector for external supply, table for a tested SAW substrate and/or fixation elements of the probe to a stationary mounted SAW substrate, indicator and connector for an external monitor. The case provides optical, electromagnetic, radiation, acoustic, vibration, anti-shock and climatic (humidity, pressure, temperature) protection of the probe.

\section{Indication}

The well-known ways and facilities to control SAW articles indicate results in the form of amplitude and phase profiles of a SAW substrate vibrations (like to those shown in Figs 2a,b). More informative are twocoordinate ways of indication - amplitude-phase hodograph and delay group time normalized by the amplitude $( \pm 1)$ and phase $( \pm \pi / 2)$. The indicated closed figure wears as a circle, if the substrate and wave on it are uniform, or as an ellipse, when the substrate has nonuniformities; defects of the substrate are demonstrated in the hodograph as short labels.

\section{Conclusion}

The compact probe for SAW performed as a "tester" provides researches of properties and control over the manufacturing technologies for series and designed SAW substrates.

\section{References}

1. R.M. De la Rue. Heterodyne optical probing of SAW in a partial standing wave situationy// IEEE Trans., SU 24(6), p. 407 - 411.

2. Filters based on surface acoustic waves (Design, technology and application). Edited by $\mathrm{M}$. Mathews. Moscow, "Radio i svyaz", 1981 (in Russian).

3. Surface acoustic waves. Edited by A.M. Oliner. Moscow, "Mir", 1981 (in Russian).

4. I.V. Blonskyi, V.G. Gryts, V.F. Kozenev, V.A. Thoryk, V.V. Semenov, Photoacoustic spectroscopy and microscopy: new diagnostic methods for materials and devices for electronics // Proceedings of SPIE 2113, p. $194-204$ (1993).

5. K.I. Britsyn, V.B. Akpambetov, V.G. Gryts, I.B. Nikitin. Visualization of SAW in acoustoelectronic facilities by using the method of optical probing // Elektronnaya tekhnika. Ser. N 10, Mikroelektronnyye ustroistva 3(15), p. 93- 96, 1979 (in Russian).

6. V.B. Akpambetov, A.D. Bzhezinskyi, K.I. Britsyn, V.G. Gryts. Investigation of band filters for SAW by using the optical probing method // Elektronnaya tekhnika. Ser. "Radiodetali $i$ komponenty" 3(60), p. 77-78, 1985 (in Russian). 